\section{(6) OPEN ACCESS}

\title{
Dysregulated activation of fetal liver programme in acute liver failure
}

\author{
Jeongeun Hyun, ${ }^{1,2}$ Seh-Hoon Oh, ${ }^{1}$ Richard T Premont, ${ }^{1}$ Cynthia D Guy, ${ }^{3}$ Carl L Berg, ${ }^{1}$ \\ Anna Mae Diehl ${ }^{1}$
}

- Additional material is published online only. To view please visit the journal online (http://dx.doi.org/10.1136/ gutjnl-2018-317603).

${ }^{1}$ Department of Medicine, Duke University, Durham, North Carolina, USA

${ }^{2}$ Regeneration Next, Duke University School of Medicine, Durham, North Carolina, USA ${ }^{3}$ Department of Pathology, Duke University, Durham, North Carolina, USA

\section{Correspondence to} Dr Anna Mae Diehl, Department of Medicine, Duke University, Durham, NC 27710, USA; annamae.diehl@duke.edu

Received 14 September 2018 Revised 3 December 2018 Accepted 20 December 2018 Published Online First 22 January 2019

\section{SLinked}

- http://dx.doi.org/10.1136/ gutjnl-2019-318218

\section{Check for updates}

(C) Author(s) (or their employer(s)) 2019. Re-use permitted under CC BY-NC. No commercial re-use. See rights and permissions. Published by BMJ.

To cite: Hyun J, Oh S-H, Premont RT, et al. Gut 2019;68:1076-1087.

\begin{abstract}
Objective Uncertainty about acute liver failure

(ALF) pathogenesis limits therapy. We postulate that ALF results from excessive reactivation of a fetal liver programme that is induced in hepatocytes when acutely injured livers regenerate. To evaluate this hypothesis, we focused on two molecules with known oncofetal properties in the liver, Yes-associated protein-1 (YAP1) and Insulin-like growth factor-2 RNA-binding protein-3 (IGF2BP3).
\end{abstract}

Design We compared normal liver with explanted livers of patients with ALF to determine if YAP1 and IGF2BP3 were induced; assessed whether these factors are upregulated when murine livers regenerate; determined if YAP1 and IGF2BP3 cooperate to activate the fetal programme in adult hepatocytes; and identified upstream signals that control these factors and thereby hepatocyte maturity during recovery from liver injury.

Results Livers of patients with ALF were massively enriched with hepatocytes expressing IGF2BP3, YAP1 and other fetal markers. Less extensive, transient accumulation of similar fetal-like cells that were proliferative and capable of anchorage-independent growth occurred in mouse livers that were regenerating after acute injury. Fetal reprogramming of hepatocytes was YAP1-dependent and involved YAP1-driven reciprocal modulation of let7 microRNAs and IGF2BP3, factors that negatively regulate each other to control fate decisions in fetal cells. Directly manipulating IGF2BP3 expression controlled the fetal-like phenotype regardless of YAP1 activity, proving that IGF2BP3 is the proximal mediator of this YAP1-directed fate.

Conclusion After acute liver injury, hepatocytes are reprogrammed to fetal-like cells by a YAP1-dependent mechanism that differentially regulates let7 and IGF2BP3, identifying novel therapeutic targets for ALF.

\section{INTRODUCTION}

Rapidly progressive liver failure (dubbed acute liver failure, ALF) can ensue after acute liver injury and cause death despite intensive medical care. However, acute injury to previously healthy livers generally causes only transient deterioration of vital liver functions and is well tolerated. ${ }^{12}$ Why dramatically divergent responses to acute liver injury occur is unknown. The explanation is not likely to be the inciting insult per se because ALF can result from drugs, toxins, viral infections and autoimmune diseases that typically cause milder, or more chronic, damage. Regardless of the inciting agent,

\section{Significance of this study}

What is already known on this subject?

- Death from acute liver failure (ALF) results from loss of vital liver functions.

- Apart from liver transplantation, liver assist devices and other interventions that aim to reconstitute these functions have generally been ineffective in reducing ALF mortality.

- Improved understanding of ALF pathogenesis is necessary to develop approaches that will restore vital liver functions in patients with ALF.

What are the new findings?

- We demonstrated that livers of patients with ALF are massively repopulated with fetalappearing hepatocytes, characterised these cells, and showed that smaller numbers of similar cells transiently accumulate in mouse livers that are regenerating effectively after acute injury.

- We discovered that the accumulation of fetallike hepatocytes in injured livers is controlled by injury-related activation of Yes-associated protein-1 and Insulin-like growth factor-2 RNAbinding protein-3, two fetal factors that are normally silenced in healthy adult hepatocytes.

- We identified mechanisms that switch the fetal programme on and off in hepatocytes.

How might it impact on clinical practice in the foreseeable future?

- This new knowledge identifies novel prognostic and therapeutic targets in ALF.

ALF histology is characterised by acute fibrosis and accumulation of cell types that are unusual in healthy livers, including progenitors and myofibroblasts. Chronic liver diseases that eventuate in cirrhosis and chronic liver failure exhibit progressive accumulation of similar cells, and patients with cirrhosis can develop an ALF-like syndrome (dubbed 'acuteon-chronic' liver failure). ${ }^{3-5}$ Acute-on-chronic liver failure is not rare: it occurs in $24 \%-40 \%$ of hospitalised patients with cirrhosis and has become a major cause of death in cirrhosis. ${ }^{34}$

Approaches to improve recovery from either type of ALF are desperately needed because liver transplantation, the only proven life-saving intervention, $^{12}$ is feasible for only a minority of patients. One solution might be to exploit the 
liver's tremendous regenerative capabilities. ${ }^{6}$ However, evidence that regeneration is defective in failing livers might make this approach challenging. Reports that recovery of some patients with acute-on-chronic liver failure was improved by transplanting adipose-derived mesenchymal stem cells or treating with cytokines that mobilise bone marrow stem cells suggest that progenitor insufficiency contributes to liver failure. ${ }^{78}$ However, contradictory data indicate that progenitor cells are overly abundant in patients with ALF $^{9}$ and show that levels of progenitor accumulation and progenitor gene expression predict acute mortality. ${ }^{1011}$ The concept that progenitor accumulation is excessive in ALF is further supported by a recent functional analysis of RNA sequencing data from mouse models of lethal and sublethal acetaminophen-induced ALF. Livers with lethal injury were dramatically enriched with transcripts encoding factors that promote fetal liver-like functions (eg, cell proliferation, development, differentiation) compared with livers with sublethal injury or healthy livers. ${ }^{12}$ Together, these findings suggested that ALF may result from dysregulation of fetal reprogramming mechanisms that are necessary for effective liver regeneration.

We propose that for the liver to regenerate effectively, some surviving liver cells must de-differentiate transiently to become more fetal-like before redifferentiating back to their mature phenotype, but that overwhelming recruitment of hepatocytes into this fetal liver-like programme, and the persistence of this state, erodes the capacity for adult liver function and results in liver failure. To evaluate this hypothesis and delineate mechanisms for fetal reprogramming in injured livers, we focused on Yes-associated protein-1 (YAP1) and Insulin-like growth factor-2 RNA-binding protein-3 (IGF2BP3) because these molecules have oncofetal properties in the liver. Both are highly expressed during fetal development, dramatically downregulated at birth and virtually undetectable in healthy adult livers, but upregulated in primary liver cancers, particularly in tumour-initiating stem-like cells, where they promote fetal-like traits. ${ }^{13-15}$ YAP1 becomes reactivated transiently in regenerating adult hepatocytes after partial hepatectomy $(\mathrm{PH}),{ }^{16}$ but it is not known if IGF2BP3 responds similarly to a regenerative challenge.

YAP1 orchestrates transcriptional responses that promote cell migration and proliferation. ${ }^{17}{ }^{18}$ In growing livers, YAP1 is gradually inactivated by Hippo kinases that are induced by cell-cell contact, ${ }^{19}$ providing a mechanism that promotes liver epithelial maturation. Conversely, constitutive activation of YAP1 in mature hepatocytes is sufficient to cause them to de-differentiate into stem-like cells. ${ }^{20}$ Constitutive YAP1 activity leads to progressive hepatomegaly and carcinogenesis after $\mathrm{PH},{ }^{21}$ indicating that YAP1 activity must be tightly regulated for an effective regenerative response. However, the mechanisms involved are unclear. IGF2BP3 is an RNA-binding protein that is critical for neoplastic and malignant transformation of mouse embryonic fibroblasts. ${ }^{22}$ IGF2BP3 regulates the localisation, stability and translation of its direct targets (ie, RNAs with IGF2BP consensus motifs), as well as numerous indirect targets that are controlled by its direct targets. Because IGF2BP3 immediately operates as a direct post-transcriptional regulator of gene expression but some of its actions ultimately impact gene transcription, IGF2BP3 dictates both immediate and longer term changes in cell fate. ${ }^{13}{ }^{23}$ This suggests that IGF2BP3 may be a conserved regulator of regenerative responses to both acute and chronic liver injury. In stem-like cells, IGF2BP3 activity is strictly constrained by the let7 family of microRNAs (miR): let7 miRs directly bind to and destabilise IGF2BP3 mRNA; conversely, IGF2BP3 protein inhibits let7 activity. ${ }^{1424}$ This mutual negative feedback loop likely explains why IGF2BP3 and let7 are reciprocally expressed in fetal livers (low let7, high IGF2BP3) and healthy adult livers (high let7, low IGF2BP3). However, it is not known if the let7-IGF2BP3 axis is involved in adult liver regeneration or whether it interacts with YAP1 to control fetal reprogramming. We examined the explanted livers of patients with ALF who required liver transplantation to determine if YAP1 and IGF2BP3 were induced; assessed whether upregulation of these oncofetal factors occurs during murine liver regeneration; determined if YAP1 and IGF2BP3 cooperate to reprogramme hepatocytes to become more fetal-like; and identified upstream signals that control these factors and thereby hepatocyte maturity during recovery from liver injury. The results identify novel diagnostic, prognostic and therapeutic targets in human ALF, an outcome of liver injury that currently is fatal in most individuals.

\section{RESULTS}

\section{Fetal reprogramming is robust in human ALF}

Loss of liver-specific function drives mortality in ALF, and fetal reprogramming is expected to suppress mature hepatocyte phenotypes. Therefore, we used immunohistochemistry to compare the expression of two factors that promote the fetal liver programme (YAP1 and IGF2BP3) in liver biopsies from a 7-year-old liver donor without known liver disease and the explanted livers of eight adult patients who required liver transplantation to survive ALF of various aetiologies (online supplementary table $\mathrm{S} 1$, figure $\mathrm{A}, \mathrm{B}$, online supplementary figures S1-S2). Although significantly younger than the adult ALF livers, the healthy child liver demonstrated negligible YAP1 or IGF2BP3. In contrast, diseased adult livers were massively repopulated with IGF2BP3-positive and YAP1-positive cells. Regenerative clusters (RC) in diseased livers were particularly enriched with such cells. These cells are strongly positive for nuclear phosphorylated SMAD2 (online supplementary figure S2B), supporting the previous results that transforming growth factor- $\beta$ signalling is increased in regenerating hepatocytes that have activated YAP1. ${ }^{25} \mathrm{RC}$ also accumulated cells expressing SOX9 and multiple cytokeratins (pan-CK), acknowledged markers of ductal liver progenitors. ${ }^{26}{ }^{27}$ In contrast, cells in RC were typically devoid of nuclear staining for CCAAT/enhancerbinding protein alpha $(\mathrm{C} / \mathrm{EBP} \alpha)$, a transcription factor expressed by mature hepatocytes ${ }^{28}$ and which was readily demonstrated in hepatocyte nuclei of the healthy child liver. Quantitative morphometry confirmed that adult ALF livers were significantly enriched with cells expressing fetal liver markers but significantly depleted of cells with mature hepatocyte markers (figure 1B, online supplementary figures S3-S5). Coimmunohistochemistry for IGF2BP3 (green) with YAP1, SOX9 or C/EBP $\alpha$ demonstrated that IGF2BP3 localised in cells which are positive for nuclear YAP1 and SOX9 but negative for $\mathrm{C} / \mathrm{EBP} \alpha$ (figure 1C). These findings suggest that human ALF develops in the context of an injury-related reprogramming process that causes surviving liver cells to acquire more fetal-like traits.

\section{Fetal reprogramming occurs transiently during effective liver regeneration in mice}

To determine if similar reprogramming occurs when regenerative mechanisms effectively reconstitute healthy adult liver parenchyma, we examined two murine models of acute liver injury in which rapid recovery of functional liver mass is thought to require regeneration of mature hepatocytes, that is, acute $70 \% \mathrm{PH}$ and acute carbon tetrachloride $\left(\mathrm{CCl}_{4}\right)$ injection. ${ }^{29-31} \mathrm{In}$ both models, we focused on time points when the replicative activity of mature hepatocytes is maximal (ie, 24-72 hours after 


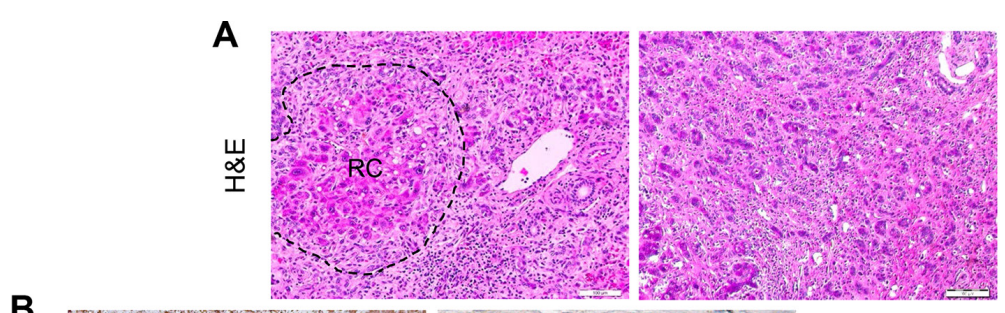

B
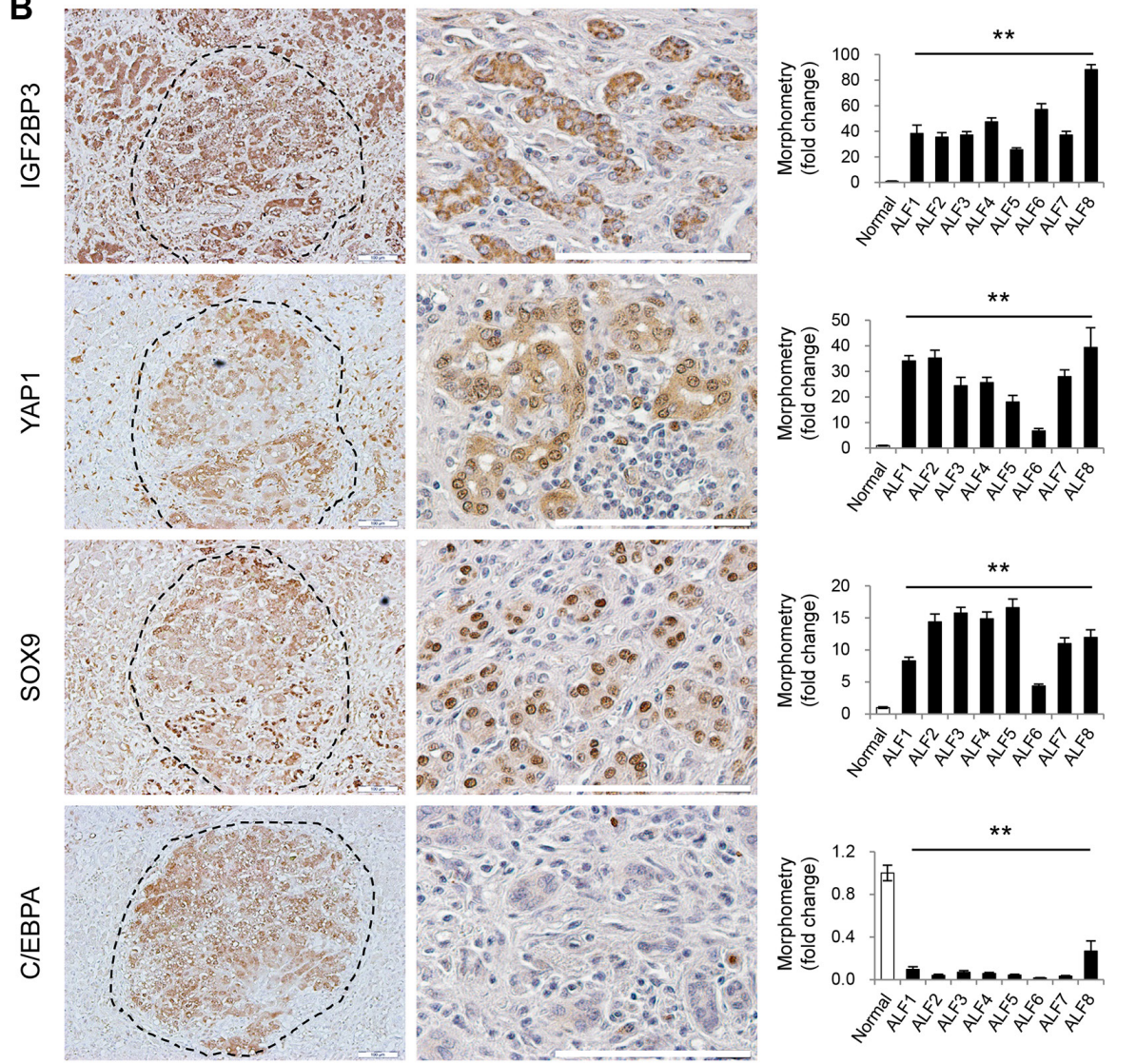

C

with YAP1

with SOX9

with C/EBPA
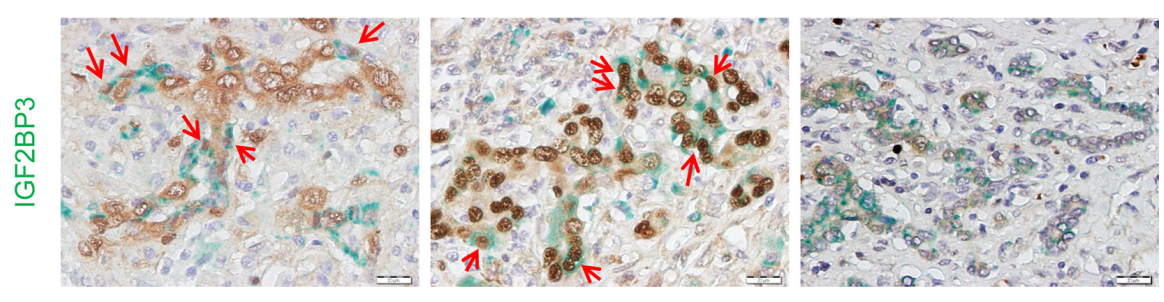

Figure 1 Hepatocytes expressing IGF2BP3 and other fetal liver cell markers accumulate in human livers with acute liver failure (ALF). (A) Representative H\&E staining in explanted liver tissues of patients with ALF. Left panel: RC, regenerating cluster (dashed line); right panel: ductalappearing cells. 100x magnification; scale bar $=100 \mu \mathrm{m}$. (B) Immunohistochemistry (IHC) for IGF2BP3, YAP1, SOX9 and C/EBP $\alpha$ in explanted liver tissues of patients with ALF. Left panels: $100 \times$ magnification; right panels: $400 \times$ magnification. Scale bar $=100 \mu m$. The mean $\pm S E M$ results from morphometric analysis are graphed and statistical analysis was performed using two-tailed Student's t-test compared with normal liver ( $\mathrm{n}=10$ $100 \times$ fields/section, ${ }^{* *} \mathrm{p}<0.005$ ). (C) Double IHC for IGF2BP3 (green) with nuclear YAP1, SOX9 or C/EBP $\alpha$ (brown). Red arrows indicate double

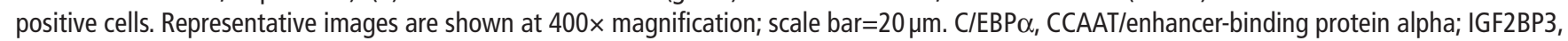
Insulin-like growth factor-2 RNA-binding protein-3.

$\mathrm{PH}$ and 2-4 days after acute $\mathrm{CCl}_{4}$ injection). Findings during the period of maximal hepatocyte regeneration were compared with those in uninjured livers (time 0 ) and with injured livers that had nearly recovered back to health (ie, livers 96 hours post-PH or 7 days post- $\mathrm{CCl}_{4}$ injection). As in healthy human liver (online supplementary figure S1B), in healthy mouse liver only rare cells expressing IGF2BP3 were demonstrated by immunohistochemistry (figure 2A). However, hepatocytic-appearing
IGF2BP3(+) cells emerged by 24 hours after PH; the number of these cells peaked at 48 hours after $\mathrm{PH}$ and then steadily declined to near basal levels by 96 hours after PH. Hepatocytes expressing IGF2BP3 also accumulated in injured areas of the livers of $\mathrm{CCl}_{4}$-injected mice (online supplementary figure S6A), and quantitative reverse transcription-PCR analysis of total liver tissues demonstrated maximal expression of $\operatorname{Ig} f 2 b p 3 \mathrm{mRNA}$ at 4 days post- $\mathrm{CCl}_{4}$ injection, the time of peak expression of 


\section{A}
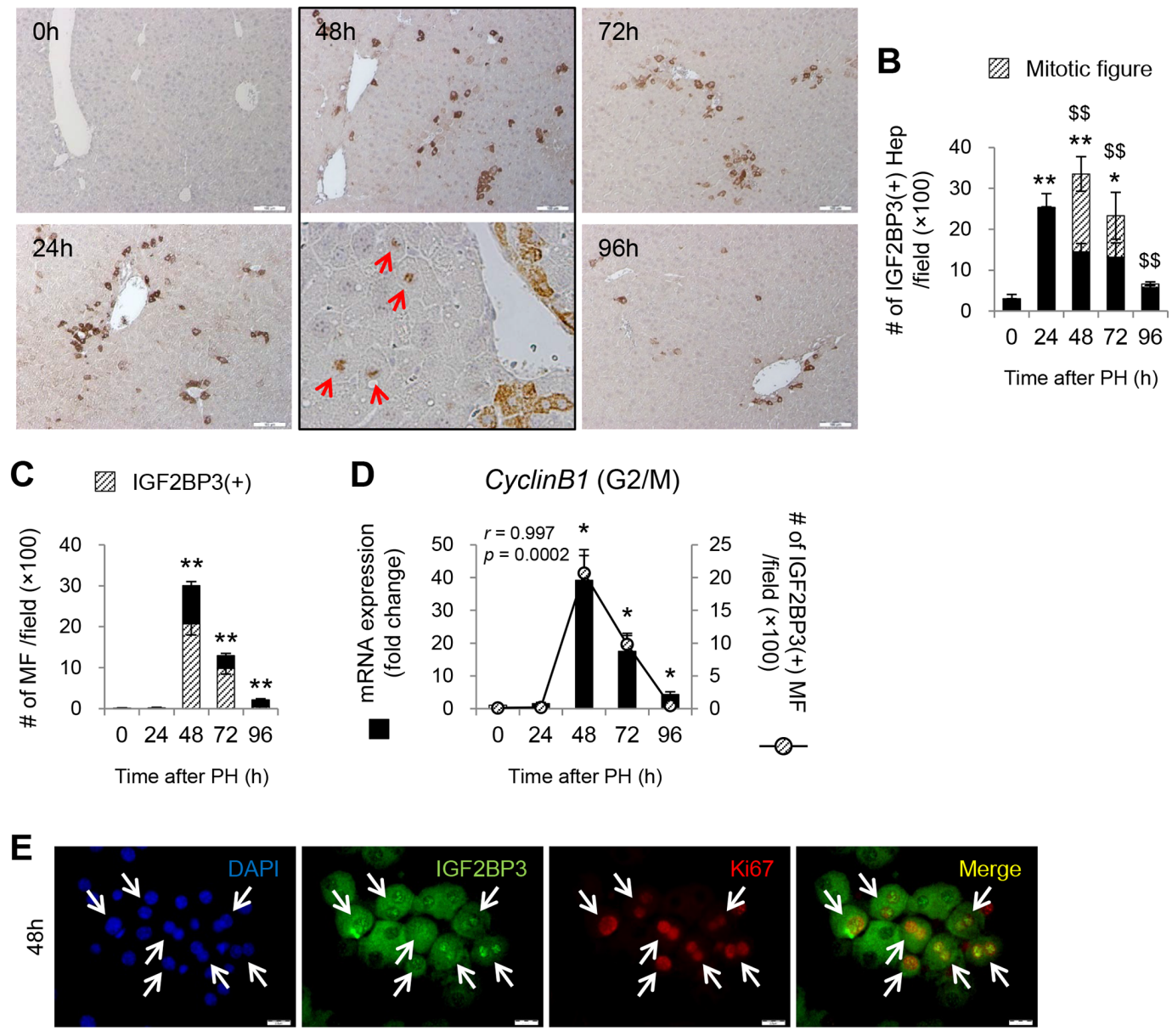

$\mathbf{F}$

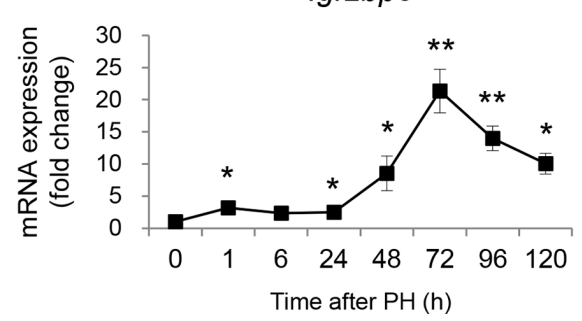

G

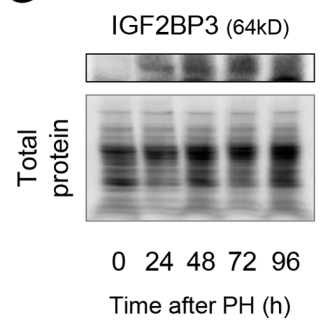

Figure 2 Effectively regenerating livers transiently accumulate proliferative IGF2BP3-positive cells. (A) Immunohistochemistry for IGF2BP3 in mouse liver sections obtained at the time of $70 \%$ partial hepatectomy (PH) (0 hour) or at 24 hours, 48 hours, 72 hours or 96 hours after $\mathrm{PH}$. Representative images are shown. Scale bar $=100 \mu \mathrm{m}$. High magnification image of 48 hours post-PH liver shows that IGF2BP3 protein localises in the cytoplasm, as well as in nuclei with mitotic figure (indicated by red arrows). (B) The number of IGF2BP3-positive hepatocytic cells were counted in 19 randomly selected 100x magnification fields/section. The proportion of IGF2BP3(+) cells in mitosis is indicated by hatched marking. The mean \pm SEM results are graphed and statistical analysis was performed using two-tailed Student's t-test compared with baseline, pre-PH (0 hour) ( $n=3$ mice/time point, ${ }^{*} p<0.05$ and ${ }^{* *} p<0.005$ for total positive cells, or $\$ \$ p<0.005$ for positive mitotic figures). (C) The percentage of total mitotic hepatocytes with IGF2BP3-positive mitotic figures was derived by counting mitotic cells in 19 randomly selected 100x magnification fields/section. The mean \pm SEM results are graphed and statistical analysis was performed using two-tailed Student's t-test compared with pre-PH (0hour) liver $\left(n=3\right.$ mice/time, $\left.{ }^{* *} \mathrm{p}<0.005\right)$. (D) Bar graph shows the mean \pm SEM results of qRT-PCR analysis for the G2-M cyclin, Cyclin B1, in primary hepatocytes freshly isolated from livers of mice before (0 hour) or after PH (24, 48, 72 or 96 hours). Significance was analysed using two-tailed Student's t-test compared with baseline (0 hour) $\left(n=3-6\right.$ mice/time, ${ }^{*} p<0.05$ ). Line graph shows the mean \pm SEM of the number of IGF2BP3-positive mitotic figures at these times. The correlation between the number of IGF2BP3-positive mitotic figures and hepatocyte expression of Cyclin B1 mRNA was analysed using Pearson's $r$ ( $r$, correlation coefficient). (E) Double immunofluorescence staining for IGF2BP3 (green) with a cell proliferation marker, Ki67 (red), in hepatocytes isolated at 48 hours post-PH. Nuclear counterstaining was done by DAPI (blue) and merged images are shown. Scale bar=20 $\mu \mathrm{m}$. (F) qRT-PCR analysis for lgf2bp3 in primary hepatocytes isolated from mice pre-PH (0 hour) or post-PH (1, 6, 24, 48, 72, 96 or 120 hours). The mean \pm SEM results are graphed and statistical analysis was performed using two-tailed Student's $\mathrm{t}$-test compared with baseline, pre-PH (0 hour) ( $\mathrm{n}=3-6 \mathrm{mice} /$ time, ${ }^{*} p<0.05,{ }^{* *} p<0.005$ ). (G) Immunoblot for IGF2BP3 normalised to total protein in lysates of primary hepatocytes freshly isolated from livers of mice at designated time points after PH. Representative blots are shown among three independent blots with similar results. DAPI, 4',6-diamidino-2phenylindole; IGF2BP3, Insulin-like growth factor-2 RNA-binding protein-3; MF, mitotic figure; qRT-PCR, quantitative reverse transcription-PCR. 
the $\mathrm{G} 2 / \mathrm{M}$ phase cyclin, Cyclin B1, indicating peak hepatocyte proliferation in that model (online supplementary figure $\mathrm{S} 6 \mathrm{~B}$ ). Morphometric analysis showed that the IGF2BP3(+) area highly correlated with the number of mitotic hepatocytes after $\mathrm{CCl}_{4}$-induced injury (online supplementary figure $\mathrm{S} 6 \mathrm{C}$ ). We had previously reported that YAP1 transiently accumulates in hepatocyte nuclei after $\mathrm{PH}$, also peaking during the replicative period before subsiding to nearly undetectable levels as regeneration is completed. ${ }^{16}$ Thus, the kinetics of IGF2BP3 induction parallels that of YAP1 activation in regenerating hepatocytes. YAP1 is known to promote liver growth after PH. ${ }^{15}{ }^{32}$ We found that many IGF2BP3 $(+)$ hepatocytes underwent mitosis from 48 to 72 hours post-PH (figure 2B). More remarkably, two-thirds or more of all the mitotic cells at these time points expressed IGF2BP3 (figure 2C) and the numbers of IGF2BP3(+) mitotic figures correlated almost perfectly with the expression of Cyclin $B 1$ (figure 2D). Further, primary hepatocytes expressing nuclear IGF2BP3 coexpressed Ki67, a cell proliferation marker, at 48 hours post-PH when nuclear Ki67 expression peaks in the hepatocyte compartment (figure 2E). As observed in regenerating livers after $\mathrm{CCl}_{4}$-induced injury (online supplementary figure S6B), the number of hepatocytes expressing IGF2BP3 and Ki67 significantly correlated in livers regenerating after $\mathrm{PH}$ $(r=0.665, p=0.018) .{ }^{25}$ Hence, expansion of the IGF2BP3(+) population likely explains the transient increase in $\operatorname{Igf} 2 b p 3$ mRNA (figure $2 \mathrm{~F}$ ) and protein expression (figure $2 \mathrm{G}$ ) that we observed in primary hepatocyte isolates that were harvested from 24 to 72 hours post-PH. Taken together, these results indicate that proliferative hepatocytes in regenerating livers are marked by expression of two oncofetal factors, IGF2BP3 and YAP1.

In addition to being more proliferative than mature hepatocytes, fetal hepatocytes differ from mature hepatocytes with regard to expression of liver progenitor markers and capacity for anchorage-independent growth. ${ }^{33}$ Therefore, we compared these parameters in primary hepatocyte isolates that were harvested from mice after sham surgery (time 0) or $\mathrm{PH}$. Compared with time 0 hepatocytes, regenerating hepatocytes expressed higher levels of several progenitor markers (eg, Fn14, Afp, Cd133, Sox9), but lower levels of mRNAs and miRs that mark mature hepatocytes, including C/ebpa and multiple let7 miR family members (figure $3 \mathrm{~A}-\mathrm{C}$ ). Interestingly, we found that primary hepatocytes freshly isolated from livers of healthy adult mice before $\mathrm{PH}$ expressed much higher levels of $42 \mathrm{kD}$ $\mathrm{C} / \mathrm{EBP} \alpha(\mathrm{p} 42)$ than the $30 \mathrm{kD}$ isoform (p30) of $\mathrm{C} / \mathrm{EBP} \alpha$. After $\mathrm{PH}$, the level of $\mathrm{p} 42 \mathrm{C} / \mathrm{EBP} \alpha$ was transiently downregulated in primary hepatocytes ( 24 hours and 72 hours post-PH) and then recovered ( 96 hours post-PH) as liver regeneration ended (figure 3C). These results are consistent with previous publications. ${ }^{35}$ In 1993, it was reported that the p42:p30 ratio increases in hepatocytes during liver development and that p30 C/EBP $\alpha$ is not antimitotic, while full-length $\mathrm{p} 42 \mathrm{C} / \mathrm{EBP} \alpha$ inhibits cell proliferation. ${ }^{36} \mathrm{~A}$ more recent study in acute myeloid leukaemia cells showed that $\mathrm{p} 30 \mathrm{C} / \mathrm{EBP} \alpha$ induces transcription of oncogenic long non-coding RNA, UCA1 (which sustains cell proliferation), whereas $\mathrm{p} 42 \mathrm{C} / \mathrm{EBP} \alpha$ represses UCA1 transcription. ${ }^{37}$ Thus, the aggregate data suggest that after $\mathrm{PH}$, the $\mathrm{C} / \mathrm{EBP} \alpha$ expression profile of adult hepatocytes becomes more fetal-like and proproliferative. Progenitor marker enrichment was also demonstrated in livers regenerating from acute $\mathrm{CCl}_{4}$-induced liver injury (online supplementary figure S7A,B). Further, when cultured for 7 days under conditions for anchorage-independent growth, hepatocyte isolates from regenerating livers formed 12 times more, and significantly larger, non-adherent spheroids than hepatocyte isolates from non-regenerating livers (time 0) (figure 3D-E). Hepatocyte isolates from regenerating livers were heterogeneous, however: some cells formed spheroids, while others became adherent to the culture plate despite exposure to conditions that favoured anchorage-independent growth. Selective analysis of these two subpopulations demonstrated that expression of the mature hepatocyte marker, C/ebpo, was relatively enriched in adherent cells. In contrast, expression of Igf2bp3 and Cd133 (fetal liver markers) was many fold higher in the spheroids. Spheroids, but not adherent cells, also expressed telomerase reverse transcriptase (Tert), a marker that distinguished regenerative from non-regenerative hepatocytes in recent lineage tracing studies ${ }^{38}$ (figure $3 F, G$ ). The aggregate data indicate that subpopulations of hepatocytes in regenerating livers become more proliferative and acquire distinctive traits of fetal liver cells.

\section{IGF2BP3 and YAP1 cooperate to reprogramme hepatocytes to become more proliferative and fetal-like}

The mechanisms responsible for reprogramming adult hepatocytes to acquire a fetal-like phenotype in regenerating livers are unknown but must be delineated in order to appropriately constrain the process and improve liver function in patients with ALF. Because our data consistently link IGF2BP3 with the fetal liver programme, we evaluated the effects on the fetal liver programme after giving small interfering (si) RNA to suppress IGF2BP3 expression in human liver cancer cells with high endogenous expression of IGF2BP3 (Hep3B cells). Results were compared with Hep3B cells that were similarly treated with non-targeting siRNA or cultured without treatment. Knocking down IGF2BP3 significantly suppressed mRNA and protein expression of AFP, SOX9 and Cyclin B1, and decreased anchorage-independent growth (figure $4 \mathrm{~A}-\mathrm{C}$ ). Conversely, treating healthy primary mouse hepatocytes (which barely express IGF2BP3) with IGF2BP3 complementary DNA to overexpress IGF2BP3 significantly increased SOX9 expression and repressed expression of $\mathrm{C} / \mathrm{EBP} \alpha$ (figure 4D). Interestingly, overexpression of IGF2BP3 had similar effects on Hep3B cells, despite their high endogenous expression of IGF2BP3 (figure 4D-E). These results reveal that IGF2BP3 can directly regulate fate decisions in non-malignant adult hepatocytes, causing these cells to become more fetal-like and proliferative, attributes that it also promotes in malignant liver cells.

During liver development, hepatocyte maturation is characterised by differential expression of RNA-binding proteins. Unlike IGF2BP3 which is downregulated in terminally differentiating hepatocytes, the RNA splicing factor, epithelial splicing regulatory protein-2 (ESRP2), is induced. Preventing ESRP2 induction during liver development promotes hepatocyte proliferation and arrests hepatocyte maturation, presumably by sustaining the expression of ESRP2-regulated targets that inhibit hepatocyte differentiation, such as YAP1. ${ }^{39}$ Although $\mathrm{PH}$ is known to induce YAP1 in adult hepatocytes, ${ }^{16}$ and our data indicate that regenerating hepatocytes become more fetal-like (figures 2-3), whether these cells suppress ESRP2 is not known. Therefore, we tracked ESRP2 expression in primary hepatocytes harvested at different times after $\mathrm{PH}$ and discovered that ESRP2 mRNAs decrease rapidly to $\sim 550 \%$ basal levels and do not recover until $\sim 120$ hours post- $\mathrm{PH}$ (figure $5 \mathrm{~A}$ ). Reciprocal changes in IGF2BP3 expression occur during this time interval (figure $2 \mathrm{~F}$ ), and immunohistochemistry confirmed that proliferative hepatocytes expressed IGF2BP3 but not ESRP2 at 48 hours post- $\mathrm{PH}$, whereas non-proliferative hepatocytes in time 0 livers were ESRP2-positive and IGF2BP3-negative (figures 2 
A Fn14

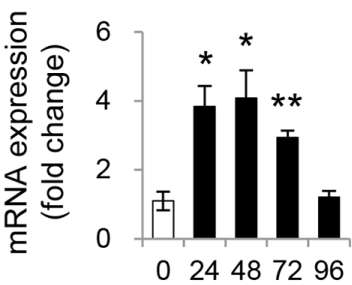

Time after $\mathrm{PH}(\mathrm{h})$
Afp

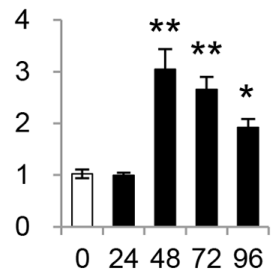

Time after $\mathrm{PH}(\mathrm{h})$
Cd133

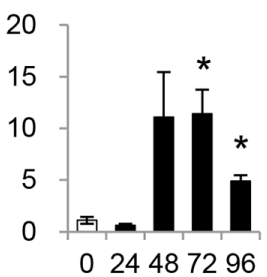

Time after $\mathrm{PH}(\mathrm{h})$
Sox 9

C/ebp $\alpha$

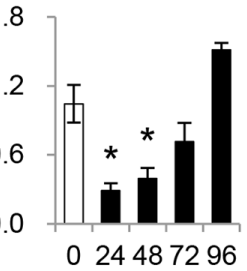

Time after $\mathrm{PH}(\mathrm{h})$
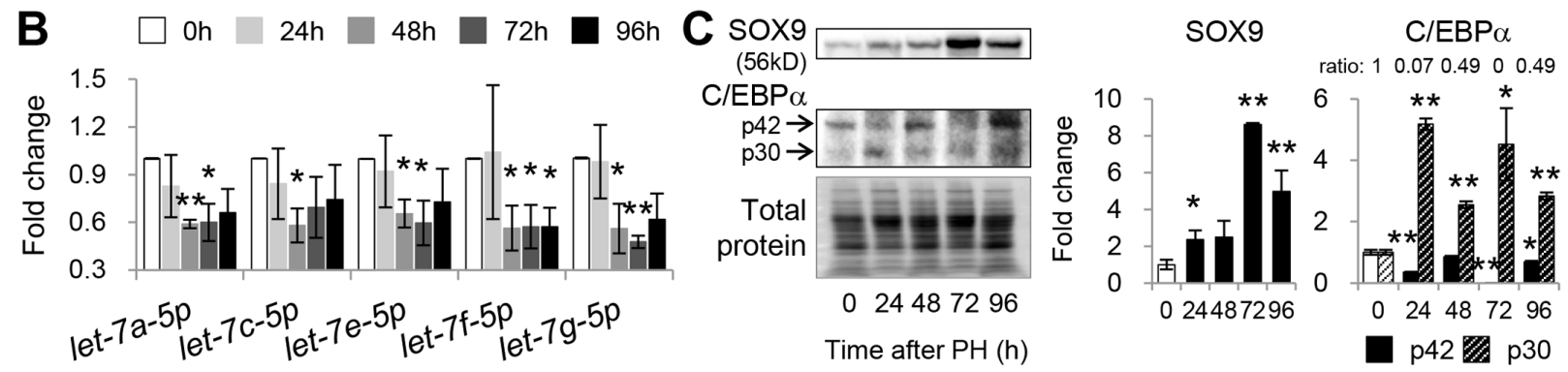

\section{D}

Day 0

Day 1

Day 4

Day 7

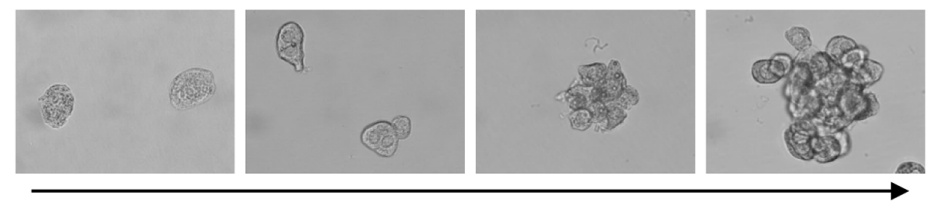

Culture under non-adherent condition $(5,000$ cell/well in 6 -well plate $)$

F

F $\quad \operatorname{lgf2bp3}$

Tert

Cd133

C/ebp $\alpha$
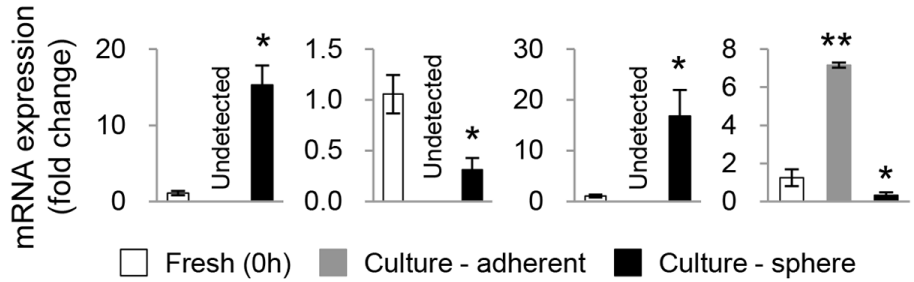

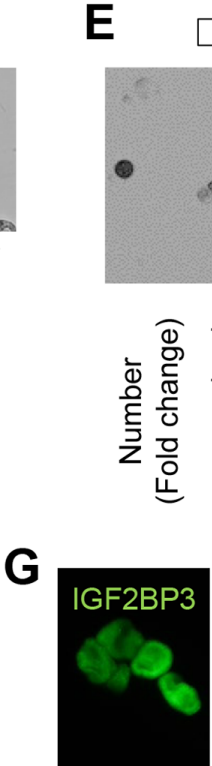

Oh

$48 h$
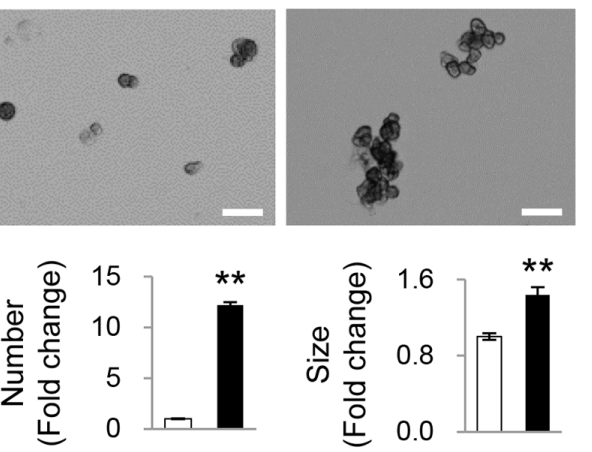

Figure 3 Regenerating hepatocytes exhibit fetal liver cell-like traits. (A and B) qRT-PCR analysis for (A) fetal liver/progenitor-cell markers including Fn14, Afp, Cd133 and Sox9, and a marker of mature hepatocytes, C/ebp $\alpha$, and (B) let7 microRNAs (let-7a-5p, let-7c-5p, let-7e-5p, let-7f-5p and let$7 g-5 p)$ in primary hepatocytes freshly isolated from livers of mice pre-PH (0 hour) or post-PH $(24,48,72$ or 96 hours). The mean \pm SEM results are graphed and statistical analysis was performed using two-tailed Student's t-test compared with baseline, pre-PH ( 0 hour) ( $n=3-6$ mice/time, * $p<0.05$, ${ }^{* *} \mathrm{p}<0.005$ ). (C) Immunoblots for SOX9 and C/EBP $\alpha$ (p42 and p30 isoforms) in protein lysates of primary hepatocytes freshly isolated from livers of mice at designated time points after PH. Representative blots are shown among three independent blots with similar results. The mean \pm SEM results of band densitometry normalised to total protein levels are displayed and statistical analysis was performed using two-tailed Student's t-test compared with 0 hour ( $n=3-6$ mice/time, $\left.{ }^{*} p<0.05,{ }^{*} p<0.005\right)$. (D and E) Sphere formation assay used primary hepatocytes isolated from mice pre-PH (0 hour) or 48 hours post- $\mathrm{PH}$. Representative micrographs show spheroids created by quiescent (0 hour) hepatocytes (E) or regenerating hepatocytes (48hours post-PH) (D and E) during 7 days of culture. The relative number and size of spheroids were analysed after 7 days of culture using the ImageJ software. The mean \pm SEM results from triplicate experiments are graphed and two-tailed Student's t-test was used to determine significance of differences versus pre-PH hepatocytes ( 0 hour) $\left(n=3\right.$ repeats/time point, $\left.{ }^{* *} p<0.005\right)$. Scale bar $=50 \mu \mathrm{m}$. (F) qRT-PCR analysis for Igf2bp3, Tert, Cd133 and C/ebp $\alpha$ in primary hepatocytes freshly isolated from mouse livers pre-PH (0 hour) or from either adherent hepatocytes or spheroid-forming free-floating hepatocytes which were cultured under non-adherent condition for 7 days after isolation from mice at 48 hours post-PH. The mean \pm SEM results are graphed. Two-tailed Student's t-test was used to determine significance of differences between 48 hours post-PH hepatocytes and hepatocytes isolated pre-PH (0 hour) ( $n=3$ repeats/group, $\left.{ }^{*} p<0.05,{ }^{*} p<0.005\right)$. (G) Representative images of double immunofluorescence staining for IGF2BP3 (green) and CD133 (red) in cytospin samples of free-floating spheroids. Nuclei were counterstained with DAPI (blue) and the merged image is shown. C/EBP $\alpha$, CCAAT/enhancer-binding protein alpha; DAPI, 4',6-diamidino-2-phenylindole; IGF2BP3, Insulinlike growth factor-2 RNA-binding protein-3; PH, partial hepatectomy; qRT-PCR, quantitative reverse transcription-PCR. 

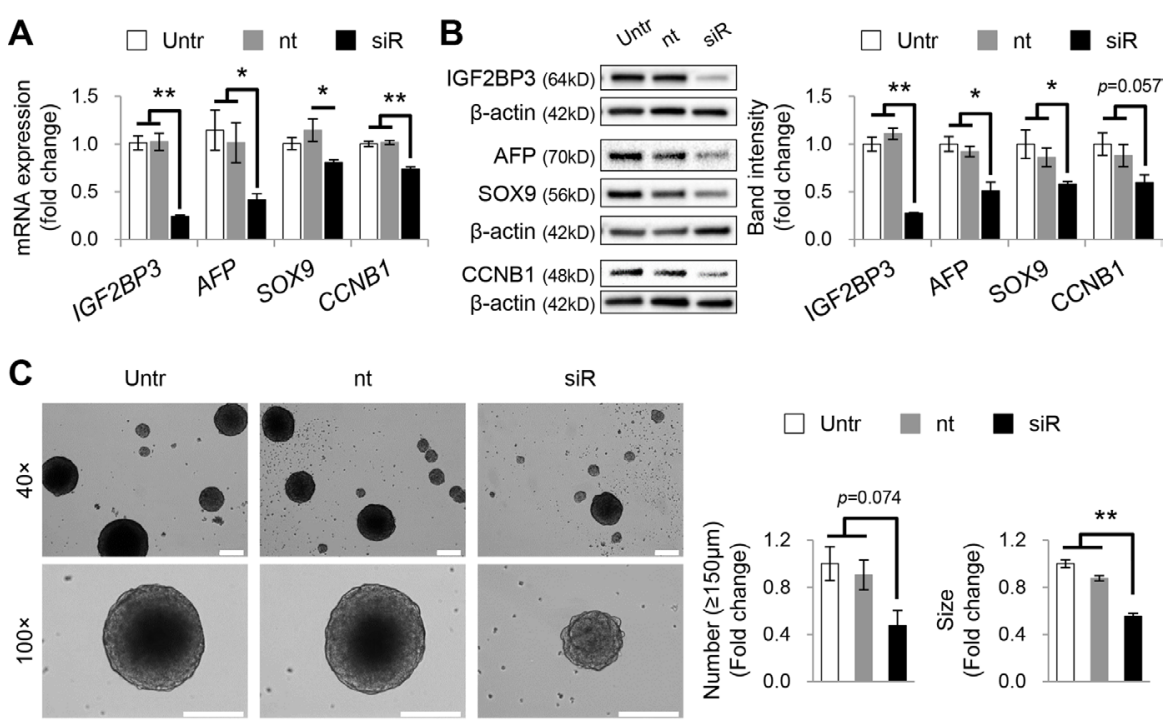

$\mathrm{nt}$
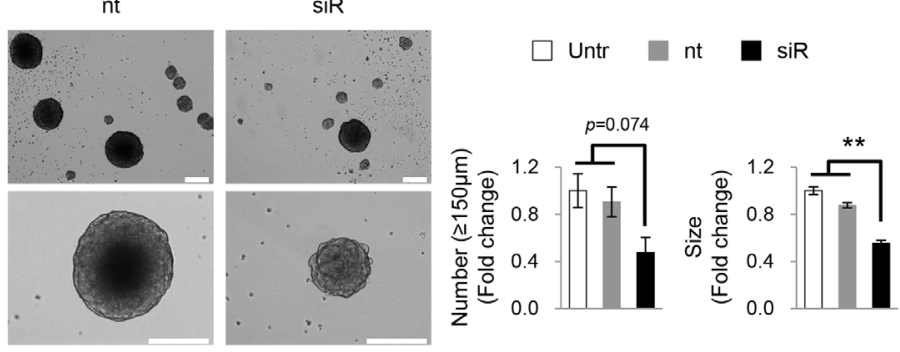

D
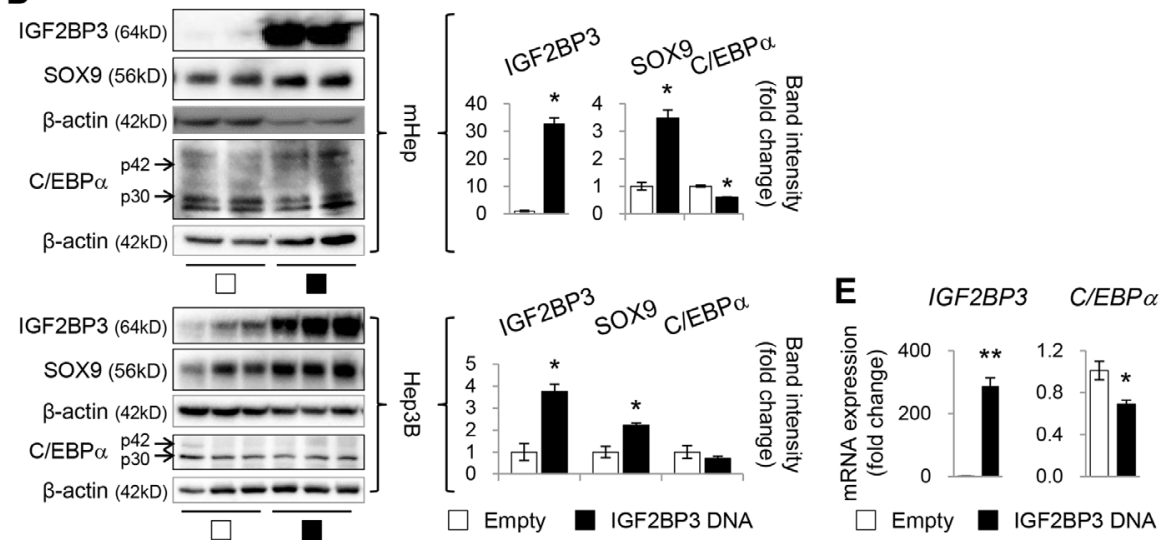

Figure 4 Fetal liver cell-like phenotype is controlled by IGF2BP3. (A) qRT-PCR analysis for IGF2BP3, AFP, SOX9 and Cyclin B1 (CCNB1) in the Hep3B human hepatoblastoma cell line, untreated (untr) or transfected with $10 \mathrm{nM}$ of IGF2BP3 siRNA (siR) or same concentration of non-targeting (nt) siRNA for 96 hours. The mean \pm SEM results from four unique experiments are graphed. Statistical analysis was performed using one-way ANOVA with Tukey corrections ( $n=4$ repeats/group, $\left.{ }^{*} p<0.05,{ }^{*} p<0.005\right)$. (B) Immunoblots for IGF2BP3, AFP, SOX9, CCNB1 and $\beta$-actin. Blots representative of three or four independent blots are shown. The mean \pm SEM results of band densitometry normalised to $\beta$-actin on all blots are graphed and statistical significance assessed using ANOVA with Tukey corrections. (C) Sphere formation assay in Hep3B untreated or transfected with either nt or IGF2BP3 siR $(10 \mathrm{nM})$. The number and size of spheres were analysed after 14 days of culture. The mean \pm SEM results from triplicate experiments are graphed and ANOVA with Tukey corrections was used to compare significance of results in treated group with untreated or non-targeting transfection groups ( $n=3$ repeats/group, ${ }^{* *} p<0.005$ ). Representative images are shown. Upper panel: $40 \times$ magnification; lower panel: $100 \times$ magnification; scale bar $=250 \mu \mathrm{m}$. (D) Immunoblots for IGF2BP3, SOX9, C/EBP $\alpha$ (p42 and p30 isoforms) and $\beta$-actin in mouse primary hepatocytes (mHep) or Hep3B transfected with $0.5 \mu \mathrm{g} /$ well of empty vector (Empty) as a negative control or pDESTmyclGF2BP3 vector (IGF2BP3 DNA) in six-well plate for 48 hours. The mean \pm SEM results of band densitometry normalised to $\beta$-actin are graphed and two-tailed Student's $t$-test was used to compare IGF2BP3 DNA cells with cells treated with empty vector ( $\mathrm{n}=3$ repeats/group, ${ }^{*} \mathrm{p}<0.05$ ). (E) qRT-PCR analysis for IGF2BP3 and C/EBP $\alpha$ in Hep3B transfected with either Empty or IGF2BP3 DNA for 48 hours. The mean \pm SEM results from triplicate experiments are graphed. Statistical analysis was performed using

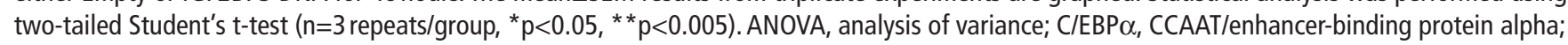
IGF2BP3, Insulin-like growth factor-2 RNA-binding protein-3; qRT-PCR, quantitative reverse transcription-PCR; siRNA, small interfering RNA.

and 5C). Western blot analysis (figure 5B) and immunohistochemistry (figure 5C) demonstrated the expected upregulation of YAP1 in regenerating hepatocytes from 24 to 72 hours post$\mathrm{PH}$, and further analysis of the western blot data (figure $2 \mathrm{~F}$ and figure 5B) confirmed that hepatocyte expressions of YAP1 and IGF2BP3 were strongly correlated $(r=0.8843, p=0.0464)$. Published results prove that YAP1 activation reprogrammes adult hepatocytes to become more proliferative and fetal-like, ${ }^{20}$ but it is not known if YAP1 and IGF2BP3 interact or operate in parallel to affect such reprogramming. Our data demonstrate that YAP1 and IGF2BP3 are coinduced in the hepatocytes of livers that are acutely failing (figure 1 , online supplementary figure S1 and S2), as well as in effectively regenerating mouse livers (figures 2 and 5, online supplementary figures S6-S7). Therefore, we evaluated how hepatocyte-specific depletion of Yap1 impacted fetal reprogramming by injecting healthy adult Yap $1^{\text {flox/flox }}$ mice with adeno-associated viral vectors that selectively introduced Cre recombinase into mature hepatocytes (AAV8-TBG-Cre) 6 days before $\mathrm{PH}$, and evaluated regenerative responses at 48 hours after PH. Unlike Yap $1^{\text {flox/flox }}$ mice that were comparably treated with control vectors (AAV8-TBG-luciferase) and thus demonstrated no Cre staining in hepatocytes, AAV8TBG-Cre-treated Yap $1^{\text {flox/flox }}$ mice had extensive Cre staining in hepatocyte nuclei both at the time of PH (time 0 ) and 48 hours 
A

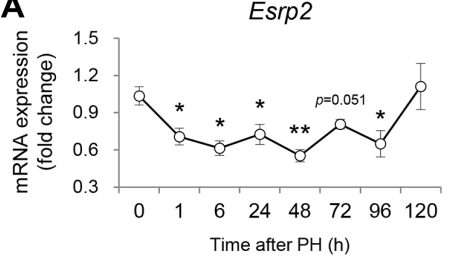

B

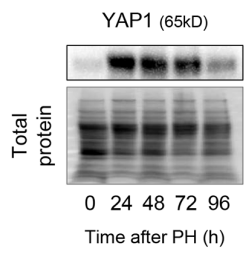

C

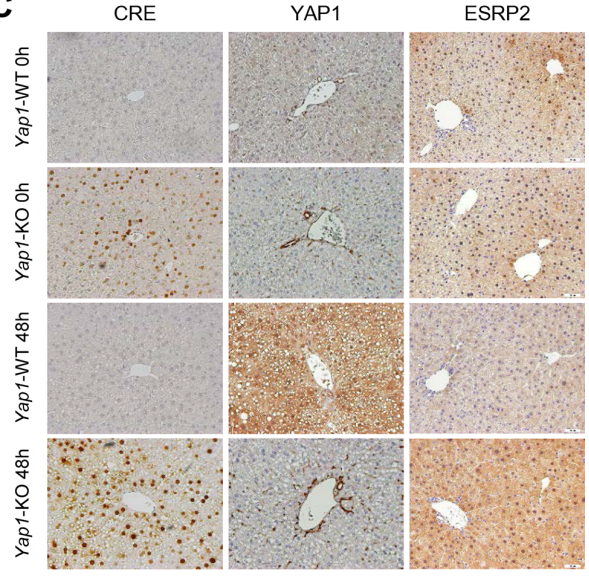

\section{D}

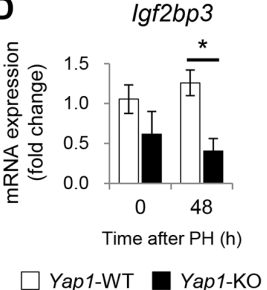

E

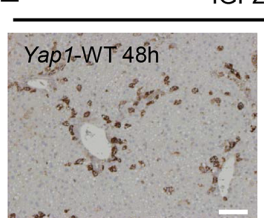

IGF2BP3

Yap1-KO 48h

F

Hepatocytes (48h post-PH)
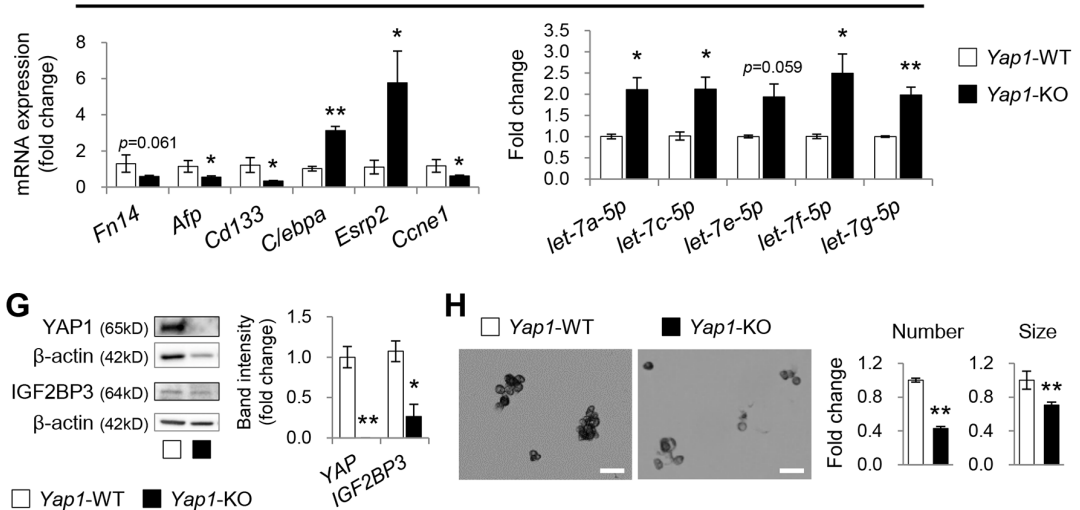

H
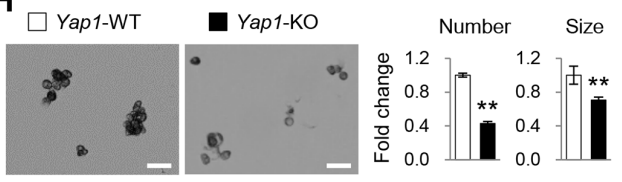

Figure 5 Targeted depletion of YAP1 in hepatocytes inhibits accumulation of IGF2BP3-positive cells and prevents livers from becoming fetal-like after a regenerative challenge. (A) qRT-PCR analysis for Esrp2 in primary hepatocytes isolated from mice pre-PH (0 hour) or post-PH $(1,6,24,48,72$, 96 or 120 hours). The mean \pm SEM results are graphed and statistical analysis was performed using two-tailed Student's t-test compared with baseline, pre-PH (0 hour) ( $n=3-6$ mice/time, ${ }^{*} p<0.05$, $\left.{ }^{* *} p<0.005\right)$. (B) Immunoblot for YAP1 normalised to total protein in lysates of primary hepatocytes freshly isolated from livers of mice at designated time points after PH. Representative blots are shown among three independent blots with similar results. (C) Yap $1^{\text {floxfflox }}$ mice were injected with either AAV8-TBG-Luciferase (Luc, Yap1-WT) or AAV8-TBG-Cre recombinase (Cre, Yap1-KO), and livers were harvested either immediately pre-PH (0 hour) or at 48 hours post-PH. Representative images of liver sections analysed for Cre recombinase, YAP1 and ESRP2 expression are shown. Scale bar=50 $\mu \mathrm{m}$. (D) qRT-PCR analysis for Igf2bp3 in liver tissues of Yap1-WT or Yap1-KO mice before or after PH. The mean \pm SEM results are graphed and significance was analysed using two-tailed Student's $\mathrm{t}$-test compared with each baseline (0 hour) or between AAV8-TBG-Luc and AAV8-TBG-Cre groups at each time point ( $n=4-7$ mice/group/time, ${ }^{*} \mathrm{p}<0.05$ ). (E) Immunohistochemistry for IGF2BP3 in liver sections of Yap1-WT or Yap1-KO mice sacrificed at 48 hours post-PH. Representative images are shown. Scale bar $=100 \mu \mathrm{m}$. The number of IGF2BP3positive hepatocytes was counted in five randomly selected 10x magnification fields/section. The proportion of IGF2BP3-positive cells with a mitotic figure is shown with hatched marking. The mean \pm SEM results are graphed and statistical analysis was performed using two-tailed Student's t-test ( $\mathrm{n}=4$ mice/group, ${ }^{*} \mathrm{p}<0.05$ for total positive cells). (F) qRT-PCR analysis for Fn14, Afp, Cd133, C/ebpo, Esrp2, Cyclin E1 (Ccne1) and let7 microRNAs in primary hepatocytes freshly isolated from livers of Yap1-WT or Yap1-KO mice at 48 hours post-PH. The mean \pm SEM results are graphed and twotailed Student's t-test was used to compare Yap1-KO with Yap1-WT ( $\mathrm{n}=4$ mice/group, ${ }^{*} \mathrm{p}<0.05$, ${ }^{* *} \mathrm{p}<0.005$ ). (G) Immunoblots for YAP1, IGF2BP3 and $\beta$-actin in protein lysates of primary hepatocytes freshly isolated from livers of Yap1-WT or Yap1-KO mice at 48 hours after PH. Representative blots are shown among three independent blots with similar results. The mean \pm SEM results of band densitometry normalised to $\beta$-actin for all blots are displayed and statistical significance was assessed using two-tailed Student's t-test $\left(n=4\right.$ mice/group, $\left.{ }^{*} p<0.05,{ }^{* *} p<0.005\right)$. (H) Sphere formation assay was performed with primary hepatocytes isolated from Yap1-WT or Yap1-KO mice at 48 hours post-PH. Representative micrographs show spheroids created by regenerating hepatocytes isolated from designated groups during 7 days of culture. The relative number and size of spheroids were analysed after 7 days of culture using the ImageJ software. The mean \pm SEM results from triplicate experiments are graphed and two-tailed Student's $t$-test was used to assess statistical significance $\left(n=3\right.$ repeats/group, $\left.{ }^{* *} p<0.005\right)$. Scale bar $=50 \mu \mathrm{m}$. C/EBP $\alpha$, CCAAT/enhancer-binding protein alpha; ESRP2, epithelial splicing regulatory protein-2; IGF2BP3, Insulin-like growth factor-2 RNA-binding protein-3; PH, partial hepatectomy; qRT-PCR, quantitative reverse transcription-PCR; YAP1, Yes-associated protein-1. 
post-PH (figure $5 \mathrm{C}$ ). These results confirmed the targeting specificity of our approach and proved that healthy mature hepatocytes are able to survive Yap1 depletion. Importantly, compared with Yap1-sufficient livers, Yap1-deficient livers expressed less Igf $2 b p 3$ mRNA both before and after PH (figure 5D). Consistent with this result, depleting Yap1 before PH blocked accumulation of IGF2BP3 $(+)$ hepatocytes, inhibited mitotic activity, markedly suppressed induction of progenitor genes, and attenuated suppression of hepatocyte differentiation markers, including ESRP2, after PH (figure 5C,E, online supplementary figure S8). The negative effects of hepatocyte-specific deletion of Yap1 on post-PH fetal reprogramming were even more apparent when we compared fetal traits in primary hepatocyte isolates from Yap1-depleted and Yap1-sufficient livers. Yap1 depletion significantly suppressed the expression of progenitor markers and reduced Cyclin E1, but enhanced expression of factors that mark mature hepatocytes, including C/ebpo, Esrp2 and multiple let7 family members (figure 5F). Western blot analysis showed that knocking down Yap1 essentially eliminated YAP1 protein in hepatocytes and reduced protein expression of IGF2BP3 by $\sim 74 \%$ (figure $5 \mathrm{G}$ ). Importantly, these Yap1/IGF2BP3-depleted hepatocytes exhibited significantly reduced capacity for anchorage-independent growth (figure $5 \mathrm{H}$ ). Together with evidence that the fetal programme is also effected by directly manipulating IGF2BP3 (figure 4), the Yap1 knockdown experiments demonstrate for the first time that two oncofetal proteins, YAP1 and IGF2BP3, cooperate with each other to reprogramme subpopulations of hepatocytes to become more proliferative and fetal-like when acutely injured livers regenerate. Because our analysis of livers of patients with ALF suggests that fetal reprogramming is overly exuberant in ALF (figure 1), it is critical to clarify what controls YAP1 and IGF2BP3 accumulation during liver repair.

\section{Central role of Let7 miRs in regulating hepatocyte accumulation of YAP1 and IGF2BP3}

The discovery that Yap1 depletion reciprocally regulates let7 and IGF2BP3 in mature hepatocytes is potentially important because the latter two factors inhibit each other in many stem-like cells. ${ }^{1424}$ Therefore, to clarify the relationship between YAP1, let7 and IGF2BP3 in liver cells, we treated Hep3B cells with different doses of verteporfin, a pharmacological inhibitor of YAP1 activity. ${ }^{40}$ Dose-dependent and time-dependent suppression of YAP1 and IGF2BP3 by verteporfin mirrored its reciprocal dose-dependent and time-dependent induction of multiple let7 family members (figure 6A-D, online supplementary figure S9A), supporting a model whereby YAP1 activity reciprocally regulates the relative abundance of an RNA-binding protein that promotes the fetal programme in hepatocytes (eg, IGF2BP3) and miRs that suppress this fetal programme to promote hepatocyte maturation (eg, let7s). Consistent with this concept, verteporfin-related inhibition of YAP1 caused a dose-dependent and time-dependent loss of fetal liver traits in Hep3B cells (figure 6E-G, online supplementary figure S9B,C). Remarkably, simply overexpressing IGF2BP3 in verteporfin-treated (ie, YAP1-inactivated) cells was sufficient to rescue the fetal programme (figure $6 \mathrm{H}$ ), proving that IGF2BP3 directly mediates some of the ultimate actions of YAP1. Further, when IGF2BP3 expression was restored in Hep3B cells, expression of YAP1 protein increased, while that of ESRP2 declined, suggesting that IGF2BP3 may induce YAP1 partially by suppressing ESRP2, an RNA splicing factor that typically downregulates YAP1 as liver development ends. ${ }^{39}$ Demonstration of multiple IGF2BP3 binding sites in
ESRP2 mRNA by in silico analysis supports this concept (online supplementary figure S10A). Importantly, hepatocyte accumulation of IGF2BP3 and YAP1 in the livers of patients with ALF associates with striking loss of ESRP2 from hepatocyte nuclei (online supplementary figure S10B), suggesting that IGF2BP3 may also interact with ESRP2 to regulate YAP1 in non-malignant adult hepatocytes. The aggregate data reveal novel post-transcriptional mechanisms that can affect rapid and critical alterations in the phenotype of adult hepatocytes (figure 6I), and thus have potential implications for diseases that occur when mature hepatocyte function is lost, including acute and acute-on-chronic liver failure.

\section{DISCUSSION}

Mortality in human liver failure is reliably predicted by algorithms that reflect loss of normal hepatic capacity for bilirubin detoxification, clotting factor biosynthesis and homeostatic regulation of other organs. Indeed, the severity of liver failure as estimated by these scoring systems establishes priority for liver transplantation. ${ }^{41}$ However, because liver transplantation is not feasible for many patients with liver failure, alternative treatments for ALF and acute-on-chronic liver failure are desperately needed. The present study raises the possibility that liver failure might be mitigated by interventions that promote hepatocyte differentiation. Our work confirms earlier reports that indicated that relatively large numbers of immature/fetal-like cells accumulate in the livers of patients with ALF. ${ }^{9-1142}$ Importantly, we also demonstrate that hepatic enrichment with immature cells is a normal characteristic of regenerating livers. Subpopulations of hepatocytic cells with fetal liver cell markers and growth characteristics emerge as mouse livers regenerate after $70 \% \mathrm{PH}$ or acute $\mathrm{CCl}_{4}$-induced liver injury. These immature liver cells derive from mature hepatocytes, since surviving hepatocytes are the source of repopulating hepatocytes in these contexts. ${ }^{29} 43$ Typically, the de-differentiation process is tightly constrained because both the numbers of immature hepatocytes that accumulate and the duration of time during which enrichment with immature hepatocytic cells persists are strictly limited following a regenerative stimulus evoked by $\mathrm{PH}$ or acute treatment with $\mathrm{CCl}_{4}$. These new data are consistent with evidence that liver dysfunction is typically mild and transient after these insults. Hence, we conclude that mechanisms that modulate the differentiation (ie, plasticity) of hepatocytes in adult livers become operative during effective liver regeneration, but are dysregulated such that hepatocyte de-differentiation predominates in failing livers, resulting in life-threatening loss of vital hepatocyte-specific functions as the liver attempts to repair.

Our studies also identified tractable mechanisms that regulate hepatocyte plasticity to control the extent of hepatocyte de-differentiation that occurs during liver regeneration. In particular, we discovered that the process is regulated by transient reactivation of mechanisms that regulate hepatocyte differentiation during liver development. More specifically, regenerating adult livers induce expression of an RNA-binding protein, IGF2BP3, which is highly expressed in fetal hepatocytes and liver stem-like cells, but normally silenced in healthy adult livers. Conversely, they suppress the expression of miRs that are prevalent in healthy adult hepatocytes but are typically rare in fetal hepatocytes (eg, let7 family members). The pattern of differential changes in IGF2BP3 and let7 content is consistent with published information indicating that these factors are mutually antagonistic, that is, IGF2BP3 inhibits let7 and vice versa. ${ }^{14} 24$ Further, the reciprocal change in hepatocyte content of IGF2BP3 

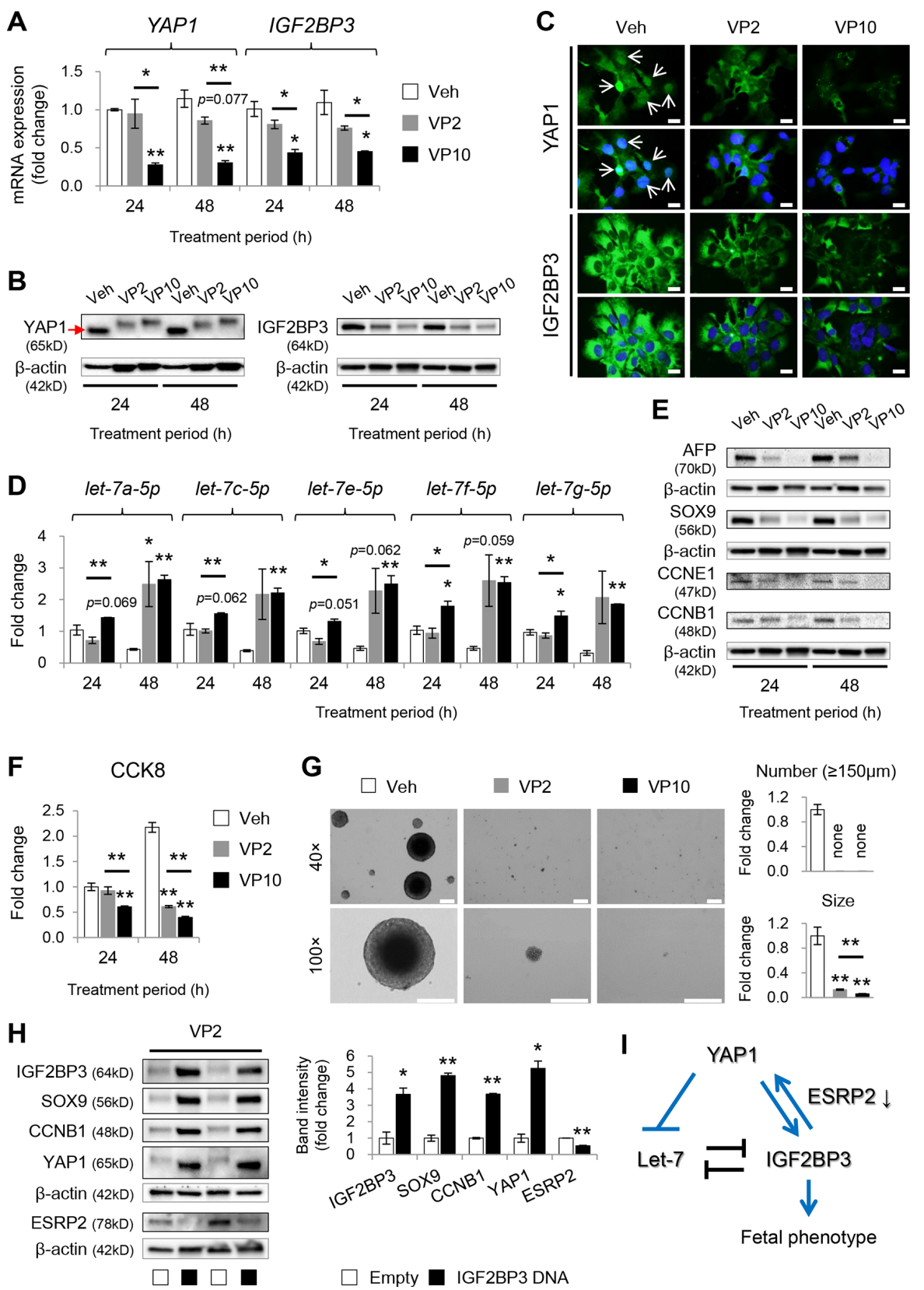

Figure 6 YAP1 and IGF2BP3 regulate each other during fetal programming. (A) qRT-PCR analysis for YAP1 and IGF2BP3 in Hep3B treated for 24 or 48 hours with 2 or $10 \mu \mathrm{M}$ of a pharmacological inhibitor of YAP1 activity, verteporfin (VP), or $0.1 \%$ DMSO as a vehicle control (Veh). (B) Immunoblots for YAP1, IGF2BP3 and $\beta$-actin in Veh-treated or VP-treated Hep3B (2 or $10 \mu \mathrm{M})$. Representative blots are shown among three independent blots with similar results. The mean \pm SEM results of band densitometry of all blots are shown in online supplementary figure S9. (C) Immunofluorescence staining for YAP1 and IGF2BP3 (green) in Hep3B treated with Veh or VP (2 or $10 \mu \mathrm{M})$ for 24 hours. White-coloured arrows indicate the nuclear localisation of YAP1. Nuclei were counterstained with DAPI (blue). Representative images are shown. Scale bar $=20 \mu \mathrm{m}$. (D) qRT-PCR analysis for let$7 a, c, e, f, g-5 p$ in Veh-treated or VP-treated Hep3Bs. All qRT-PCR data are graphed as mean \pm SEM results from triplicate experiments ( $\mathrm{n}=3$ repeats/ group/time, $\left.{ }^{*} p<0.05,{ }^{* *} p<0.005\right)$. (E) Immunoblots for AFP, SOX9, CCNE1, CCNB1 and $\beta$-actin in Veh-treated or VP-treated Hep3B. Representative blots are shown among three independent blots with similar results. The mean \pm SEM results of band densitometry for all blots are displayed in online supplementary figure S9C. (F) Cell viability/proliferation was assessed by Cell Counting Kit-8 (CCK8) assay in Hep3B treated with either Veh or VP. The mean \pm SEM results are graphed ( $n=6$ repeats/group/time, $\left.{ }^{*} p<0.005\right)$. (G) Sphere formation assay in Hep3B treated with Veh or VP $(2$ or $10 \mu M)$. The number and size of spheres were analysed after 14 days of culture. The mean \pm SEM results from triplicate experiments are graphed ( $n=3$ repeats/ group, $\left.{ }^{* *} \mathrm{p}<0.005\right)$. Representative images are shown. Upper panel: $40 \times$ magnification; lower panel: $100 \times$ magnification; scale bar $=250 \mu \mathrm{m}$.

Statistical significance of all differences was assessed using two-tailed Student's t-test. (H) Immunoblots for IGF2BP3, SOX9, CCNB1, YAP1, ESRP2 and $\beta$-actin in Hep3B treated with $2 \mu \mathrm{M}$ of VP for 24 hours 1 day after treatment with either empty vector (Empty) or pDESTmyclGF2BP3 vector (IGF2BP3 DNA). Representative blots are shown among three independent blots with similar results. The mean $\pm S E M$ results of band densitometry for all blots are displayed, and statistical analysis was assessed using two-tailed Student's t-test compared with empty vector-treated Hep3B ( $n=3$ repeats/group, ${ }^{*} p<0.05,{ }^{* *} p<0.005$ ). (I) Putative model of post-transcriptional mechanisms regulating the phenotype of adult hepatocytes by YAP1, ESRP2, let7 and IGF2BP3. DAPI, 4',6-diamidino-2-phenylindole; DMSO, dimethyl sulfoxide; IGF2BP3, Insulin-like growth factor-2 RNA-binding protein-3; qRT$\mathrm{PCR}$, quantitative reverse transcription-PCR; YAP1, Yes-associated protein-1. 
RNA-binding protein and let7 miRs that follows a regenerative challenge has the predicted consequence, that is, de-differentiation of adult hepatocytes to a more immature phenotype. These results have exciting clinical implications because they suggest that replenishing let7s might restore hepatocyte maturity in failing livers, given that let7s inhibit IGF2BP3 and we showed that IGF2BP3 is a proximal effector of the fetal-like phenotype in hepatocytes. Appropriate evaluation of this issue will be challenging, but the strategy seems promising based on an earlier publication that reported that exogenous expression of let7 was sufficient to abrogate the growth of murine liver cancers that depend on stem-like IGF2BP3(+) tumour-initiating cells. ${ }^{44}$ Finally, we uncovered a previously unsuspected relationship between YAP1 and the let7-IGF2BP3 axis. This discovery in adult hepatocytes may have broad implications because YAP1 activation antagonises epithelial differentiation in many tissues, including the liver. ${ }^{20}{ }^{45} 46$ In our studies, hepatocyte-specific deletion of Yap1 blocked both suppression of let7 and induction of IGF2BP3 and led to inhibited hepatocyte de-differentiation and proliferation. Further, overexpressing IGF2BP3 in verteporfin-treated (YAP1-inactivated) hepatoblastoma cells restored the expression of YAP1 and YAP1-inducible genes that mark proliferative fetal-like cells (eg, SOX9, CCNB1) while suppressing the expression of ESRP2, an RNA splicing factor that typically inhibits YAP1, suppresses hepatocyte proliferation and promotes hepatocyte maturation at the end of liver development. ${ }^{39}$ These novel results identify YAP1 as both a regulator and a target of the let7-IGF2BP3 axis and thus suggest that inhibiting YAP1 activation may be another approach to reverse hepatocyte de-differentiation and restore hepatic function in ALF.

In summary, effective regeneration of fully functional hepatic parenchyma following liver injury requires replacement of dead hepatocytes with healthy mature hepatocytes. This process necessitates precise regulation of hepatocyte plasticity so that progenitor populations are mobilised but also replenished. Loss of vital hepatocyte-specific functions, that is, liver failure, ensues when the process becomes biased to favour accumulation of immature hepatocytes. Mechanisms that programme cells to be stem-like/progenitor-cell like during fetal development are reactivated, and then silenced, in hepatocytes during effective regenerative responses. Manipulating the factors that drive fetal programming, including YAP1, IGF2BP3 and let7, is able to restrict accumulation of immature cells in injured livers, identifying these factors and the pathways they regulate as novel therapeutic targets to improve recovery from liver failure.

\section{MATERIALS AND METHODS \\ Experimental animal model}

Male, adult C57BL/6J wild-type (WT) mice (Jackson Laboratory, Bar Harbor) ( $\mathrm{n}=42)$ and Yap $1^{\text {flox/flox }}$ mice (from Dr Udayan Apte, University of Kansas) ( $\mathrm{n}=21$ ) underwent $\mathrm{PH}$ or sham surgery. Hepatocytes or livers were harvested $0,24,48,72$ or 96 hours later. Male Yap $1^{\text {floxflox }}$ mice were injected via the tail vein with $5 \times 10^{11}$ genome equivalents of AAV8-TBG-Luc or AAV8-TBG-Cre (University of Pennsylvania Viral Vector Core) 6 days before PH. Twelve additional WT mice were sacrificed $0,2,4$ or 7 days after a single injection of $\mathrm{CCl}_{4}(0.753 \mathrm{~mL} / \mathrm{kg}$ dissolved in olive oil). ${ }^{47}$

\section{Human liver samples}

Formalin-fixed paraffin-embedded sections of the normal liver $(n=1)$ and explanted livers of patients with ALF $(n=8)$ were obtained from the Duke Pathology Archives and analysed with MetaMorph Software (Molecular Devices).

\section{Statistics}

Results are expressed as mean \pm SEM and analysed by two-tailed Student's t-test or one-way analysis of variance, followed by a post-hoc Tukey's test. Significant differences were $p<0.05$. All correlation analyses were analysed by Pearson's correlation coefficient (r).

Contributors $\mathrm{JH}$ : helping with the design of the research studies, conducting the experiments, acquiring data, analysing data, providing reagents, writing the manuscript. S-HO: conducting the experiments, acquiring the data. RTP: conducting the experiments, acquiring the data. CDG: providing human liver samples. CLB: providing human liver samples. AMD: designing the research studies, analysing the data, writing the manuscript, securing funding for the research.

Funding This work was supported by NIH grants DK106633, AA010154 and DK077794, the Florence McAlister Professorship of Medicine (AMD) and an award from the Duke Regeneration Next Initiative (JH).

\section{Competing interests None declared.}

\section{Patient consent for publication Not required.}

Ethics approval Animal studies were conducted under an approved Duke University IACUC protocol as per the National Research Council 'Guide for the Care and Use of Laboratory Animals'. Human livers were obtained and analysed in accordance with a Duke Institutional Review Board-approved protocol (Pro00087196)

Provenance and peer review Not commissioned; externally peer reviewed.

Open access This is an open access article distributed in accordance with the Creative Commons Attribution Non Commercial (CC BY-NC 4.0) license, which permits others to distribute, remix, adapt, build upon this work non-commercially, and license their derivative works on different terms, provided the original work is properly cited, appropriate credit is given, any changes made indicated, and the use is non-commercial. See: http://creativecommons.org/licenses/by-nc/4.0/.

\section{REFERENCES}

1 Bernal W, Wendon J. Acute Liver Failure. N Engl J Med Overseas Ed 2013;369:2525-34.

2 Lee WM, Squires RH, Nyberg SL, et al. Acute liver failure: Summary of a workshop. Hepatology 2008;47:1401-15.

3 Hernaez R, Solà E, Moreau R, et al. Acute-on-chronic liver failure: an update. Gut 2017;66:541-53.

4 Jalan R, Gines P, Olson JC, et al. Acute-on chronic liver failure. J Hepatol 2012;57:1336-48.

5 Sarin SK, Choudhury A. Acute-on-chronic liver failure: terminology, mechanisms and management. Nat Rev Gastroenterol Hepatol 2016;13:131-49.

6 Taub R. Liver regeneration: from myth to mechanism. Nat Rev Mol Cell Biol 2004;5:836-47.

7 Garg V, Garg H, Khan A, et al. Granulocyte colony-stimulating factor mobilizes CD34(+) cells and improves survival of patients with acute-on-chronic liver failure. Gastroenterology 2012;142:505-12.

8 Chen G, Jin Y, Shi X, et al. Adipose-derived stem cell-based treatment for acute liver failure. Stem Cell Res Ther 2015;6:40.

9 Dubuquoy L, Louvet A, Lassailly G, et al. Progenitor cell expansion and impaired hepatocyte regeneration in explanted livers from alcoholic hepatitis. Gut 2015:64:1949-60.

10 Jung Y, Brown KD, Witek RP, et al. Accumulation of hedgehog-responsive progenitors parallels alcoholic liver disease severity in mice and humans. Gastroenterology 2008;134:1532-43.

11 Sancho-Bru P, Altamirano J, Rodrigo-Torres D, et al. Liver progenitor cell markers correlate with liver damage and predict short-term mortality in patients with alcoholic hepatitis. Hepatology 2012;55:1931-41.

12 Li C, Ming Y, Hong W, et al. Comparison of hepatic transcriptome profiling between acute liver injury and acute liver failure induced by acetaminophen in mice. Toxicol Lett 2018;283:69-76.

13 Bell JL, Wächter K, Mühleck B, et al. Insulin-like growth factor 2 mRNA-binding proteins (IGF2BPs): post-transcriptional drivers of cancer progression? Cell Mol Life Sci 2013;70:2657-75

14 Degrauwe N, Suvà ML, Janiszewska M, et al. IMPs: an RNA-binding protein family that provides a link between stem cell maintenance in normal development and cancer. Genes Dev 2016;30:2459-74.

15 Yimlamai D, Fowl BH, Camargo FD. Emerging evidence on the role of the Hippo/YAP pathway in liver physiology and cancer. J Hepatol 2015;63:1491-501. 
16 Swiderska-Syn M, Xie G, Michelotti GA, et al. Hedgehog regulates yes-associated protein 1 in regenerating mouse liver. Hepatology 2016;64:232-44.

17 Lian I, Kim J, Okazawa H, et al. The role of YAP transcription coactivator in regulating stem cell self-renewal and differentiation. Genes Dev 2010;24:1106-18.

18 Zhao B, Ye X, Yu J, et al. TEAD mediates YAP-dependent gene induction and growth control. Genes Dev 2008;22:1962-71.

19 Zhao B, Wei X, Li W, et al. Inactivation of YAP oncoprotein by the Hippo pathway is involved in cell contact inhibition and tissue growth control. Genes Dev 2007;21:2747-61.

20 Yimlamai D, Christodoulou C, Galli GG, et al. Hippo pathway activity influences liver cell fate. Cell 2014;157:1324-38.

21 Benhamouche S, Curto M, Saotome I, et al. Nf2/Merlin controls progenitor homeostasis and tumorigenesis in the liver. Genes Dev 2010;24:1718-30.

22 JnBaptiste CK, Gurtan AM, Thai KK, et al. Dicer loss and recovery induce an oncogenic switch driven by transcriptional activation of the oncofetal Imp1-3 family. Genes Dev 2017;31:674-87.

23 Jønson L, Christiansen J, Hansen TV, et al. IMP3 RNP safe houses prevent miRNAdirected HMGA2 mRNA decay in cancer and development. Cell Rep 2014;7:539-51.

24 Kugel S, Sebastián C, Fitamant J, et al. SIRT6 suppresses pancreatic cancer through control of lin28b. Cell 2016;165:1401-15.

25 Oh SH, Swiderska-Syn M, Jewell ML, et al. Liver regeneration requires Yap1TGF $\beta$-dependent epithelial-mesenchymal transition in hepatocytes. J Hepatol 2018;69:359-67.

26 Kawaguchi Y. Sox9 and programming of liver and pancreatic progenitors. J Clin Invest 2013;123:1881-6.

27 Lu WY, Bird TG, Boulter L, et al. Hepatic progenitor cells of biliary origin with liver repopulation capacity. Nat Cell Biol 2015;17:971-83.

28 Shiojiri N, Takeshita K, Yamasaki H, et al. Suppression of C/EBP alpha expression in biliary cell differentiation from hepatoblasts during mouse liver development. $J$ Hepatol 2004;41:790-8.

29 Xu W, Hellerbrand C, Köhler UA, et al. The Nrf2 transcription factor protects from toxin-induced liver injury and fibrosis. Lab Invest 2008;88:1068-78.

30 Yu C, Wang F, Jin C, et al. Increased carbon tetrachloride-induced liver injury and fibrosis in FGFR4-deficient mice. Am J Pathol 2002;161:2003-10.

31 Michalopoulos GK. Liver regeneration. J Cell Physiol 2007;213:286-300.

32 Grijalva JL, Huizenga M, Mueller K, et al. Dynamic alterations in Hippo signaling pathway and YAP activation during liver regeneration. Am J Physiol Gastrointest Liver Physiol 2014;307:G196-G204.
$33 \mathrm{Ma} \mathrm{S}$, Chan KW, Hu L, et al. Identification and characterization of tumorigenic liver cancer stem/progenitor cells. Gastroenterology 2007;132:2542-56.

34 Kitade M, Factor VM, Andersen JB, et al. Specific fate decisions in adult hepatic progenitor cells driven by MET and EGFR signaling. Genes Dev 2013;27:1706-17.

35 Jin J, Hong IH, Lewis K, et al. Cooperation of C/EBP family proteins and chromatin remodeling proteins is essential for termination of liver regeneration. Hepatology 2015;61:315-25.

36 Lin FT, MacDougald OA, Diehl AM, et al. A 30-kDa alternative translation product of the CCAAT/enhancer binding protein alpha message: transcriptional activator lacking antimitotic activity. Proc Natl Acad Sci U S A 1993;90:9606-10.

37 Hughes JM, Legnini I, Salvatori B, et al. C/EBP $\alpha-p 30$ protein induces expression of the oncogenic long non-coding RNA UCA1 in acute myeloid leukemia. Oncotarget 2015;6:18534-44.

38 Lin S, Nascimento EM, Gajera CR, et al. Distributed hepatocytes expressing telomerase repopulate the liver in homeostasis and injury. Nature 2018;556:244-8.

39 Bhate A, Parker DJ, Bebee TW, et al. ESRP2 controls an adult splicing programme in hepatocytes to support postnatal liver maturation. Nat Commun 2015;6:8768.

40 Liu-Chittenden Y, Huang B, Shim JS, et al. Genetic and pharmacological disruption of the TEAD-YAP complex suppresses the oncogenic activity of YAP. Genes Dev 2012;26:1300-5.

41 Wiesner R, Edwards E, Freeman R, et al. Model for end-stage liver disease (MELD) and allocation of donor livers. Gastroenterology 2003;124:91-6.

42 Hattoum A, Rubin E, Orr A, et al. Expression of hepatocyte epidermal growth factor receptor, FAS and glypican 3 in EpCAM-positive regenerative clusters of hepatocytes, cholangiocytes, and progenitor cells in human liver failure. Hum Pathol 2013;44:743-9.

43 Fausto N, Campbell JS. The role of hepatocytes and oval cells in liver regeneration and repopulation. Mech Dev 2003;120:117-30.

44 Nguyen LH, Robinton DA, Seligson MT, et al. Lin28b is sufficient to drive liver cancer and necessary for its maintenance in murine models. Cancer Cell 2014;26:248-61.

45 Camargo FD, Gokhale S, Johnnidis JB, et al. YAP1 increases organ size and expands undifferentiated progenitor cells. Curr Biol 2007;17:2054-60.

46 Mahoney JE, Mori M, Szymaniak AD, et al. The hippo pathway effector Yap controls patterning and differentiation of airway epithelial progenitors. Dev Cell 2014;30:137-50

47 Chen Y, Choi SS, Michelotti GA, et al. Hedgehog controls hepatic stellate cell fate by regulating metabolism. Gastroenterology 2012;143:1319-29. 